\title{
A Pattern of Economic Anaseism in Scientific and Technological Progress
}

\author{
Mikhail Grishin ${ }^{1,2, *}$ \\ ${ }^{1}$ The Russian Presidential Academy of National Economy and Public Administration, 119571 \\ Moscow, Russia \\ ${ }^{2}$ Federal Service for Hydrometeorology and Environmental Monitoring (Roshydromet), 125993 \\ Moscow, Russia
}

\begin{abstract}
The article reveals and theoretically substantiates the anaseism (wave-like) nature of scientific and technological progress. The author of the article, in the course of his research activities, has developed a model of waves (anaseism) of scientific and technological progress, which reveals a natural sequence of the life cycle of any technology, start-up and other, both tangible and intangible objects of scientific and technological progress.
\end{abstract}

\section{Introduction}

In the author's view, any technology is as much a commodity in the consumer market as any other product created through the labour energy expended by the worker. If this is true, then this means that any technology possesses the equilibrium properties of any commodity that Karl Marx wrote about, namely value properties:

1. Consumption value;

2. Exchange value.

According to Marx in his book "Capital": "Consumption value is realised only in the process of consumption. Consumption values form the substance of wealth, whatever its social form. In the type of society to be considered, they are at the same time the material bearers of exchange value. Exchange value is first of all presented as a quantitative ratio, as a proportion, in which consumption values of one kind are exchanged for consumption values of another kind" Ошибка! Источник ссылки не найден..

The above statements are confirmed in practice by applying the calculation method, with the construction of the model developed by Theodore Levitt in 1965 . The concept is based on the fact that any product will sooner or later be displaced from the market by another, better or cheaper product. In marketing, there is no such thing as a perpetual good 2.

Based on the author's assertion that any technology is a commodity, and as the quotes above suggest, that it has equilibrium properties, then like any commodity, the Lifecycle Method is applicable to technology.

\footnotetext{
* Corresponding author: diplomat009@mail.ru
} 
In the course of the research work, the features of the technology that a 'simple' or finished product as an end-use product does not possess, were identified.

Based on the material presented, such features include the so-called "exchange value", because although technology has the basic properties that are inherent in every commodity, it cannot be sold until a certain point. Unlike a product, which, in the case of an early sale, i.e. before its completion, becomes a semi-finished product or raw material, without losing its marketability (sale/purchase), but by reducing the surplus value resulting from its sale.

This concept has set the author the objective of developing a model that defines the economic properties of anaseism (waves) in scientific and technological progress.

\section{Theory}

Taking as a basis the concept of Kondratyev waves outlined in the "Great Cycles of Conjuncture and Foresight Theory. Selected Writings" on the following: "Modern economic science has a predominantly statistical nature, on the other hand, it increasingly comes to realise the need to develop a dynamic theory" 3 and using, as a fundamental basis when developing its theory, the concept of "product life cycle" described by Philip Kotler in his book Marketing Basics", which states that any product has four stages as follows:

1. market introduction stage;

2. growth stage;

3. maturity stage;

4. decline stage4.

From the above quotation, in the course of his research, the author came to the necessity of constructing anaseism (waves), in order to explain the economic processes that take place during the development and subsequent implementation of a technology with a change in its initial state from intangible to tangible.

Based on the author's thesis that technology is a commodity, a model of anaseism (waves) of scientific and technological progress (Figure 1 and 2) has been built, which allows us to trace the level of economic recovery (growth) of a particular technology over a certain time period, as well as, after the transition of technology (start-up) from intangible to tangible (commodity) state, to predict the stage of economic and technological decline of goods, as a tangible product with specific material properties, and goods as a intangible product with the properties of scientific and technological and moral deterioration.

\section{Results and Discussion}

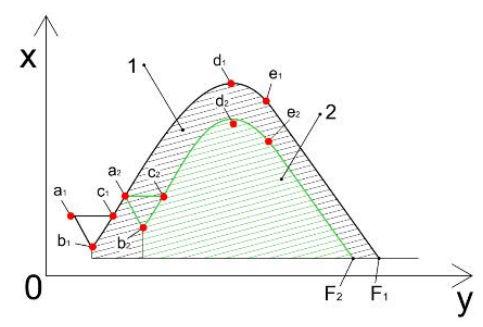

Fig. 1. Model of anaseism (waves) of scientific and technological progress: I - external waves; II - internal waves.

The model developed by the author (Figure 1) shows that there are two types of anaseism (waves) of scientific and technological progress:

I. External (core) - have the highest potential for technology implementation; 
II. Internal (auxiliary) - are based on Type I waves and are an upgrade of an already established technology.

In turn, anaseism (waves) of both types is divided into three classes (Figure 2):

1. Solid (higher) type waves (1.3 and 2.3 ) - have the most powerful potential for scientific and technological progress. This means that during the growth period $(\mathrm{c} 1,2,3 \ldots)$ and up to the point of maximum $(\mathrm{d} 1,2,3 \ldots)$, the greatest growth of both the entire scientific and technological progress and the individual sphere of activity occurs. Such waves have the highest capital returns with little initial financial investment.

2. Mesomorphic (medium) type waves (1.2 and 2.2) - these waves do not possess the properties of the solid waves, but despite this, it is the mesomorphic waves that are fundamental in the study of scientific and technological progress as these waves create the technology that does not massively change the human life, but creates a precedent for its significant transformation.

3. Hominid (lower) type waves (1.1 and 2.1) - these waves, when studying the holistic behavior of scientific and technological progress anaseism, do not have much capital output, however, they preserve the properties of type 2 and 3 waves, i.e. temporal duration. These waves are characterized by low resource intensity (costs) and highly specialized nature of the object under development.

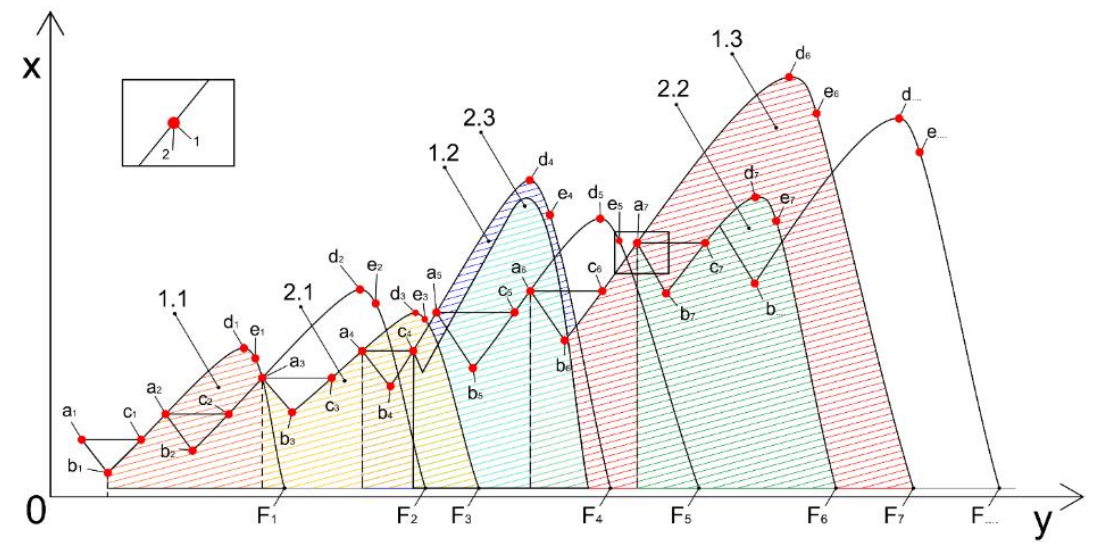

Fig 2. Model of classes of anaseism (waves) of scientific and technological progress: 1.1 and $2.1-$ Hominid (lower) type waves; 1.2 and 2.2 - Mesomorphic (medium) type waves; 1.3 and 2.3 - Solid (higher) type waves.

Designations:

1. X-axis coordinate segment - time indicator (minutes, hours, days, months, years)

2. Y-axis coordinate segment - denotes the amount of resources expended and/or received in the form of losses and/or gains (net profit).

Points $\left(a_{1,2,4} \ldots\right)$ - are the "birthplaces" of a new technology or, as in case of points $\left(a_{3,5,7} \ldots\right)$, upgrade of an existing technology.

Points $\left(b_{1,2,3} \ldots\right)$ - are the places where the technology being developed is "launched" and transformed into an object of mass consumption, i.e. into a real and tangible product with exchange value.

1. Segment from points $\left(a_{1,2}, 3 \ldots\right)$ to points $\left(b_{1,2}, \ldots\right)$ - is a temporal and quantitative indicator that reflects the material and temporal costs incurred in developing the technology and preparing it for mass (serial) production and orienting the market towards its consumption. 
2. Points $\left(c_{1,2,3} \ldots\right)$ - are the places of full recovery of those material costs that were incurred during the development and mass launch of the "new" or "improved/upgraded" S\&T facility (start-up).

3. Segment from points $\left(b_{1,2,3} \ldots\right)$ to points $\left(c_{1,2,3} \ldots\right)$ - is a temporal and quantitative indicator that reflects the profit (net profit) over a certain time period obtained after the technology has been transformed into a material and commodity state with the consumption and exchange value.

4. Points $\left(\mathrm{d}_{1,2,3 \ldots}\right)$ - are the places of the "maximum" of profit (net profit) generated by the knowledge-based technological object created).

5. Segment from points $\left(\mathrm{c}_{1,2,3} \ldots\right)$ to points $\left(\mathrm{a}_{2,3,4} \ldots\right)$ - is a temporal and quantitative indicator that reflects the profit received (net profit) over a certain time period, from $98-99 \%$ of which must be invested in a new science and technology or innovation project, in order to strengthen and expand market presence. Only 1-2\% of the net profit generated by the project can be used as an object of tangible enrichment.

6. Segment from points $\left(\mathrm{a}_{2,3,4 \ldots)}\right.$ to points $\left(\mathrm{d}_{1,2,3 \ldots}\right)$ - is a temporal and quantitative indicator that reflects the stage of economic growth (rise) of a scientific and technological object (start-up), its market sustainability and the profit (net profit) generated from its implementation. This segment (period) is characterised by the feature of a steady increase in the rate of production of a knowledge-based technological product with a continuous increase in the profit (net profit) generated.

7. Points $\left(\mathrm{e}_{1,2,3 \ldots}\right)$ - is the moment of "decline" of the resulting profit (net profit) from the knowledge-based technological product created, as an object that has consumption and exchange value.

8. Segment from points $\left(\mathrm{d}_{1,2,3 \ldots}\right)$ to points $\left(\mathrm{e}_{1,2,3 \ldots}\right)$ - is a temporal and quantitative indicator that reflects market oversaturation with a given product and, consequently, the reduction (decrease) of the resulting profit (net profit) due to a decrease in consumer purchasing power, moral and technological exhaustion of a particular product (goods).

9. Points $\left(\mathrm{F}_{1,2,3 \ldots}\right)$ - are the market "agony" points for any start-up/scientific and technological product. These points are characterized by a worldwide mass consumption of the product. The product ceases to be "unique" and becomes a mass-market necessity. There are various analogues, copies, and so on. The product ceases to bring in substantial revenue, but does not "leave" the mass-market.

10. Segment from points $\left(\mathrm{e}_{1,2,3 \ldots}\right)$ to points $\left(\mathrm{F}_{1,2,3 \ldots}\right)$ - is a temporal and quantitative indicator that reflects the market stagnation of a product (goods), as a knowledgebased object and as a product with consumption and exchange value over a specific time period.

\section{Conclusion}

In the course of the scientific research conducted by the author, it has been:

1. identified the time and cost relationship in the life cycle of a technology and its transition to a commodity as a product of consumption;

2. established and scientifically proven the continuity of the symbiotic state between technology as a commodity and the commodity as a product of the scientific and technological process;

3. The author has developed a model of the scientific and technological progress anaseism that allows making accurate and clear predictive graphs, thanks to which one can determine the level of global scientific and technological development with 
the possibility of determining to which of the two types of anaseism belongs a particular product of scientific and technological activity, a start-up or an upgrade of already existing technology and specific tangible product created on its basis.

4. The anaseism model proposed by the author makes it possible to determine at the state-strategic or transnational corporate level:

a) Whether a technology is a new object of scientific and technological progress or whether it merely improves (upgrades) an existing object of scientific and technological progress;

b) Allows strategic management to adjust and redirect activities to qualitatively change a specific technological activity, with a perspective (radically changing) the system as a whole.

\section{References}

1. K. Marx , "Capital" Critique of Political Economy, 1, 797 (1952)

2. P.E. Chernozubenko, Life cycle, Marketer Notes, http://www.marketch.ru/

3. N.D. Kondratyev, Y.V. Yakovets, L.I. Abalkin, Great cycles of conjuncture and foresight theory, Selected works, 766 (2002)

4. Kotler Philip, Kotler Marketing Basics, https://mybook.ru/ 